\title{
Probiotics and Gut Health: A Prophylactic to Disease
}

\section{Alissa Welsher*}

Scientific Manager Gut Health, USDA National Institute of Food and Agriculture, University of Arkansac, USA

*Corresponding author: Alissa Welsher, Scientific Manager Gut Health, USDA

\section{Review Article}

Volume 2 Issue 5

Received Date: November 14, 2017

Published Date: December 15, 2017

National Institute of Food and Agriculture, University of Arkansac, USA, Tel: 4128412474; E-mail: alissa.piekarski@gmail.com

\section{Abstract}

It has been discovered that about $70 \%$ or more of the immune system is located in the gastrointestinal tract, making it the largest immune organ in the body. Along these lines, the gut is the point of nutrient absorption and is constantly in flux. Probiotics and other 'holistic' type methods have gained recent popularity due, in part, to an increasing risk of allergies to certain medications and antibiotic resistance. Though the idea of microbial diversity in the gut has been around for a long time, it has not been until recently that studies have been conducted to try and delineate the mechanisms by through which they work. This review aims to discuss the main methods currently used, their benefits, and some risks that can occur when these approaches are self-dose

Keywords: Probiotic; Bacteriotherapy; Gut; Gealth

\section{Introduction}

Probiotics have long been used to prevent illness and aid in restoring gut conditions to normality, having been traced to the first use of cheese and fermented products by the ancient Greeks and Romans. The concept of ingesting microorganisms associated with benefits for humans and animals began with elie Metchnikoff in the early $20^{\text {th }}$ century. He postulated that residents of a community in Bulgaria, who ate yogurt, generally lived longer due to this custom. This led to the suggestion of "the dependence of the intestinal microbes on the food makes it possible to adopt measures to modify the flora in our bodies and to replace the harmful microbes by useful microbes" [1]. This lead to more interest in the idea of useful microbes colonizing the gut with many species discovered afterwards, each with their own benefit: Bifidobacteria, found in gut flora of infants fed breast milk, had clinical benefits for treating diarrhea; Escherichia coli used in acute gastrointestinal infectious salmonellosis and shigellosis; and Lactobacillus acidophilus transforming the gut micro flora and aiding in the relief of constipation [2]. Although the idea of probiotics has been around for a long time, it has only been recently that it has gained much attention, not only by scientists but, especially, by consumers.

Studies in humans have demonstrated that, although the possibility of having a core micro biota exists, there tend to be large variations in overall micro biota composition [3]. This can be no clearer than when observing micro biota of individuals from different populations and different environments. For example, the spread of fecal bacteria is diminishing in modern infants, particularly in western countries where excessive hygiene limits circulation, delaying establishment of the bacteria. 


\section{Food Science and Nutrition Technology}

Comparing these infants to those in countries in the Middle East and Africa, infants, even those delivered through caesarean section; acquire stable fecal populations within one week [4]. Another example show $\mathrm{s}$ that in the past decade, there has been an increase in Staphylococci (a bacterium not previously regarded as intestinal) thought to be due to a lack of competition from resident gut micro biota [4]. This phenomenon is seen very clearly in the agricultural industry as well, particularly in chickens. When a chicken is born, it is normally introduced to parental bacteria through the eggshell and surrounding nest. In the case of modern hatcheries, a very sterile environment, the chicks are left to obtain these bacteria from the world around them: workers who place them in houses, the boxes they are shipped in, and food and water in the house. The resulting macrobiotic in these animals not only varies from house to house, but between individuals chicks as well. Establishment of a host micro flora is crucial for development and immunity in all species and, recently, it has become an uphill battle to develop.

Many methods have been adopted to try and recolonize or replenish a host micro biota to establish a healthy composition. Bacteriotherapy, through fecal transplantation, has been used to treat recurrent Clostridium difficile- associated disease with resulting resolution of the symptoms. There tends to be a similarity as well, between the recipient and donor intestinal micro biota following treatment, suggesting the donor's bacteria quickly occupy niches resulting in restoration of the structure and function of the microbial communities present [5]. Another method is influencing diet through feeding of prebiotics, carbohydrate products, which help feed the beneficial bacteria in the gut. Trace elements, such as selenium, have also been found to produce similar results to prebiotics. Studies have shown that the diversity of the micro biota increased as a result of selenium in the diet [6]. When viewed, it appeared that selenium had unique effects across microbial taxa and germ-free mice fed these diets showed higher levels and activity of glutathione peroxidase 1 and methionine-Rsulfoxide reductase 1 , both known selenoproteins. Their conclusion was that dietary selenium affected both microflora composition and colonization of the gastrointestinal tract which influenced host selenium status and selenoproteome expression [6]. One more, and yet possible the most popular, are utilizing probiotics to obtain and maintain a healthy gut environment.

Probiotics are defined as live, microbial food supplements that act beneficially by improving the intestinal microbial balance of their host. Some of the more popular health benefits include: improving overall intestinal tract health, enhancing the immune system, synthesizing and enhancing the bioavailability of nutrients, decreasing the prevalence of allergies - for example, lactose intolerance, and even as far as reducing the risk of certain cancers [7]. Although much research is currently being performed on how these microbes act as prophylactics, the mechanism by which they perform these functions are largely unknown, leaving much room for scrutiny. Scientists have discovered that most of the pathways or mechanisms by which probiotic act are through modification of gut $\mathrm{pH}$, producing antimicrobial or antibacterial compounds making the environment inhospitable to other 'harmful' bacteria, competing for receptor sites, nutrients, and growth factors, stimulating the immune system, and producing lactase [7].

However, risks have also been attributed to probiotic treatments. In the case of infants and young children, Lactobacillus rhamnosus has been studied in the fight against viral infectious diarrhea and atopic eczema. Recently, there has been an increase in the incidence of allergic sensitization among children receiving $L$. rhamnosus-supplemented infant formula feed at an early age [8]. The consumer group for this formula is children below the age of 12 months and is intended for long-term daily use, even though there is not much knowledge on long-term effects on the host micro biota with large doses of live bacteria. A main reason for probiotics gain in popularity is due to the scare of antibiotic resistance not realizing that some probiotics may carry these resistance genes as well. In humans, many parents are taking a stance against standard medical practices and are more concerned about what is going into them as well as their children. Thoughts of antibiotic resistance has motivated the parents to choose a holistic approach, including probiotics in their families daily lives in the hopes that they stay healthy and can better combat future illnesses. Although probiotics have gained momentum in the prevention fight against disease, they have the potential to alter intestinal metabolism, transfer genes coding for resistance to antimicrobials, and other adjuvant effects that warrant further investigation. In live production animals, antibiotic usage as a growth promoter is becoming rarer due to bans such as the EU's and companies such as McDonalds and Tyson taking a stance to not utilize meat that has been treated with antibiotics. Due to these movements, probiotics have gained popularity among livestock integrators as a means to prevent illness in their animals and regain the trust of a community already wary with where their food comes from and how it is handled. 


\section{Food Science and Nutrition Technology}

In conclusion, methods such as bacteriotherapy, influencing diet through feed, and probiotics are meant to act more as prophylactics than treatments. Even though some have known benefits in treating certain diseases, there are not many clinical findings suggesting these were the main reasons why such disease were cured or stymied. They are meant to provide a healthy foundation on which to build a more diverse and, in turn, more robust gut, preparing it to better outcompete harmful bacteria. Having recently discovered that about $70 \%$ of the immune system resides in the gut, this may mean a healthier individual overall, but should by no means infer their use to be a sole and final treatment. In the end, it is the duty of the individual to consult with a medical doctor or nutritionist so these professionals can develop individualized dietary plans in which the benefits of probiotics and other such treatments and be fully and successfully utilized.

\section{References}

1. Metchnikoff É (1907) the prolongation of life: Optimistic studies. Springer Classics in Longevity and Aging, New York, NY Springer, pp: 116.

2. Cheplin HA, Rettger LF (1920) Studies on the Transformation of the Intestinal Flora, with Special Reference to the Implantation of Bacillus Acidophilus: II. Feeding Experiments on Man. Proceedings of the National Academy of Sciences of the United States of America 6(12): 704-705.
3. Stanley D, Geier MS, Hughes RJ, Denman SE, Moore RJ (2017) Highly Variable Microbiota Development in the Chicken Gastrointestinal Tract. Engormix 1-17.

4. Alderberth I, Lindberg E, Aberg N, Hesselmar B, Saalman R, et al. (2006) Reduced enterobacterial and increased staphylococcal colonization of the infantile bowel: an effect of hygenic lifestyle? Pediatr Res 59(1): 96-101.

5. Khoruts A, Dicksved J, Jansson J, Sadowsky MJ (2010) Changes in the Composition of the Human Fecal Microbiome After Bacteriotherapy for Recurrent Clostridium difficile-associated Diarrhea. Journal of Clinical Gastroenterology 44(5): 354-360.

6. Kasaikina MV, Kravtsova MA, Lee BC, Seravalli J, Peterson DA, et al. (2011) Dietary selenium affects host selenoproteome expression by influencing the gut microbiota. FASEB J 25(7): 2492-2499.

7. Kopp-Hoolihan (2001) Prophylactic and therapeutic uses of probiotics: a review. J Am Diet Assoc 101(2): 239-241.

8. Yazdankhah SP, Natyhus JA, Eggesbo M, Midtvedt T, lassen J, et al. (2009) Should long-term prophylactic use of probiotics for infants and young children give cause for concern? Microbial ecology in health and disease 20(4): 171-176. 